\title{
Le facteur anthropomorphe: élément d'analyse dans la communication non verbale?
}

\author{
El factor antropomorfo: ¿elemento de análisis de la comunicación \\ no verbal?
}
The anthropomorphe factor: analysis element of nonverbal communication?

\author{
Jonathan Rodríguez Jaime ${ }^{1}$ ORCID - Michel B. Cislak ${ }^{2}$ ORCID \\ ${ }^{1}$ Institución Universitaria Politécnico Grancolombiano, ${ }^{2}$ Université de Lorraine \\ Colombia - Francia
}

Fecha correspondencia: Recibido: julio 30 de 2016. Aceptado: junio 20 de 2017.

Forma de citar:

Rodríguez Jaime., J., \& Cislak, M.B. (2017). Le facteur anthropomorphe: élément d'analyse dans la communication non verbale?. Revista CES Psicol.,10(2), 126-142.

Open access

(c) Copyright

Licencia creative commons

Etica de publicaciones

Revisión por pares

Gestión por Open Journal System

DOl: http://dx.doi.org/10.21615/

cesp.10.2.9

ISSN: 2011-3080

\section{Sobrel el artículo}

* Cet article se nourrit des trouvailles réalisées dans "Aproximación al factor antropomorfo: el cuerpo como estímulo en la comunicación no verbal" écrit appartenant à la ligne de recherche en cognition social, du groupe: Psicología, Educación y Cultura PEC-PG, lequel a été effectué

Comparte

\section{Résumé}

Les mesures, les formes, les proportions et l'apparence du corps humain, c'est-a-dire, le facteur anthropomorphe (FA), a produit un désaccord académique mondial, d'une part, on retrouve des scientifiques qui l'incorporent à l'étude de la communication non verbale (CNV), tandis que d'autres auteurs l'excluent. C'est pour cette raison, que le but de cet article est d'élucider si le FA est un élément d'analyse dans la CNV. Afin de faire cela, il a été nécessaire de réaliser une épuration conceptuelle à travers un examen ontologique. Cette recherche apporte donc des critères pour créer un cadre théorique du FA comme sous-catégorie de la CNV. En examinant la doctrine scientifique et les études effectuées par la psychologie évolutionniste, il a été révélé que le FA: a) est un signe non-linguistique qui envoie au moins 15 messages différents, b) concorde avec la définition de CNV et répond à tous ses éléments structurels, c) on peut le trouver comme mécanisme d'expression dans d'autres espèces et d) permet de soutenir le système verbal en exécutant quatre fonctions, par conséquent, il est indispensable de continuer avec des études expérimentales dont la portée soit limitée à la psychologie de la communication, de sorte que, la communauté académique puisse explorer d'autres aspects du FA impliqués dans la cognition sociale.

Mots clés: Communication non Verbale, Corps Humain, Facteur Anthropomorphe, Psychologie de la Communication.

\section{Resumen}

Las medidas, formas, proporciones y apariencia del cuerpo humano, es decir, el factor antropomorfo (FA), ha generado un desacuerdo académico mundial, de un lado, están científicos que lo incorporan al estudio de la comunicación no verbal (CNV), de otro, autores que lo excluyen. Por tal razón, el propósito de este artículo es dilucidar si el FA es un elemento de análisis en la CNV. Para tal fin, fue necesaria una depuración conceptual mediante un examen ontológico, por ello, esta investigación aporta criterios para crear un marco teórico del FA como sub-categoría de la CNV. Además, al 
Pág 127

par le premier auteur afin d'obtenir le titre de psychologue. Une reconnaissance spéciale à Alice Prada Anthropologue diplômée du Master en Science Social de I'Université de Bordeaux-II pour les recommandations méthodologiques et à Emmanuelle Michaut et Kerrikay Leon pour ses commentaires d'écriture.

Sobre los autores:

1. Avocat et psychologue de l'Institución Universitaria Politécnico Grancolombiano.

2. Philologue et candidat au Master en sciences du langage de l'Université de Lorraine, France. revisar la doctrina científica e investigaciones de psicólogos evolucionistas, se encuentra que el FA: a) es un signo no lingüístico que envía al menos 15 mensajes diferentes, b) se acopla a la definición de CNV y cumple con todos sus elementos estructurales, c) es posible encontrarlo como mecanismo de expresión en otras especies $y, d)$ sirve de apoyo al sistema verbal ejecutando cuatro funciones. En consecuencia, se debe continuar con investigaciones experimentales cuyo alcance se circunscriba al área de psicología de la comunicación, para así explorar otros aspectos del FA implicados en la cognición social.

Palabras clave: Comunicación no Verbal, Cuerpo Humano, Factor Antropomorfo, Psicología de la Comunicación.

\section{Abstract}

The sizes, forms, proportions and appearance of the human body, i.e. the anthropomorphic factor (AF), has generated worldwide academic disagreement. On one side, there are scientists who include it in the study of nonverbal communication (NVC), on the other hand, there are authors who exclude it. For this reason, the purpose of this article is to elucidate whether the AF is an analytical element in NVC. To this end, a conceptual decantation was necessary through an ontological examination; therefore, this research provides criteria to create a theoretical framework of the AF as subcategory of the NVC. In addition, when reviewing scientific doctrine and research of evolutionary psychologists, it is found that the AF: a) is a non-linguistic sign that sends at least 15 different messages, b) is coupled with the definition of NVC and complies with all its structural elements, c) it can be found as a mechanism of expression in other species and d) it supports the verbal system by executing 4 four functions. Consequently, it is mandatory to continue with experimental research which is limited to communication psychology, in order to explore other AF aspects involved in social cognition.

Keywords: Nonverbal Communication, Human Body, Anthropomorphic Factor, Communication Psychology.

\section{Introduction}

La communication non verbale (CNV) est un thème particulièrement pertinent pour la recherche scientifique au cours des quatre dernières décennies, en somme, aujourd'hui de nouveaux résultats sont découverts, puisque cette discipline contribue à une variété d'aspects de l'être humain; dont l'un d'entre eux est l'exécution des relations sociales (Kwon, Ogawa, Ono, et Miyake, 2015). Pour tout ce qui précède, selon Hernández et Rodríguez (2009), il existe au moins six sciences qui analysent ce sujet: "la psychologie, la psychiatrie, l'anthropologie, la sociologie, l'éthologie et la linguistique" (p. 65, notre traduction).

Cependant, malgré la grande quantité de multiples disciplines, une grande majorité des contributions scientifiques a eu comme objet l'étude du langage corporel, ainsi, les recherches ont ségrégué un important nombre de thèmes, d'autres éléments inhérents méritent aussi un développement plus profond. Cette polarisation pourrait devenir du fait que, pour certains auteurs, la CNV peut se définir comme "n'importe quel mouvement, réflexif ou non, d'une partie ou de la totalité du corps qu'une personne emploie pour communiquer un message émotionnel au monde extérieur" (Fast, 1979, p.5, notre traduction), position partagée par Robles, Hernandez et Fajar- 
Le concept ci-dessus a été peu abordé par la psychologie de la communication et depuis cette perspective, il n'existe pas de littérature scientifique non plus. Pour cette raison, le FA ou ses composants manquent de définition explicite, en fait, dans certaines contributions académiques elles ne sont même pas encore envisagées. do (2011) et Pons (2015) qui conçoivent le langage corporel comme l'équivalent du concept communication non verbale.

En revanche, ce qui précède est une vue réductionniste, puisque, le langage du corps n'est pas synonyme exacte du mot "communication non verbale", chaque fois que, cette dernière précise de manière générale "l'échange d'informations à travers des signes non linguistiques" (Garcia, 2000, p. 21), tandis que le premier se limite à un type de signe spécifique (les gestes).

Ainsi, il est nécessaire de souligner que selon Boutaud (2004) un signe est un élément provenant d'un émetteur, qui envisage en plus un signifié pour un récepteur, pour toutes ces raisons, en dehors du langage du corps, il existe d'autres catégories de la CNV telles que: la proxémique (gestion de l'espace) (Hall, 1984), le paralangage (tons, nuances ou rythmes de la voix) (Xu, Lee, Wing-Li, Liu, et Birkholz, 2013), les vêtements (où ils sont étudiés comme des éléments d'expression de l'individualité) (Casablanca et Chacón, 2014) et enfin "l'apparence personnelle"(Leathers et Eaves, 2016, p.137, notre traduction) où se trouve "l'aspect physique et l'apparence (indices non verbaux qui restent imperturbables telles que la forme du corps)" (Hernández. et Rodríguez, 2009, p.65, notre traduction).

Néanmoins, le dernier terme est simplement énoncé par les auteurs, peut-être car sur cette question, il n'existe pas un développement théorique solide dans les articles de CNV, où soient mentionnées des études expérimentales. Son influence n'a pas non plus été évaluée dans le processus communicatif, du moins, en faisant référence à des sources académiques actualisées et fiables, sujet auquel il manque le travail de Knapp (1995) cité dans plusieurs écrits sur la CNV. De plus, concevoir "l'aspect physique" "l'apparence" ou "la forme du corps" manquent de précision. Ainsi afin d'assurer la bonne exécution de la méthodologie herméneutique ${ }^{1}$ utilisée dans cet article et en accueillant les résultats de Little, Jones et DeBruine (2011), on considère nécessaire la délimitation de cette catégorie d'analyse pour qu'elle n'inclue non seulement la forme, mais aussi d'autres dimensions du corps, ce qui, dans cet article est appelé facteur anthropomorphe (FA).

Le concept ci-dessus a été peu abordé par la psychologie de la communication et depuis cette perspective, il n'existe pas de littérature scientifique non plus. Pour cette raison, le FA ou ses composants manquent de définition explicite, en fait, dans certaines contributions académiques elles ne sont même pas encore envisagées, comme le prouvent les travaux de: Fast (1979); De Lavergne (2010); Owen (2011); Robles, Hernandez et Fajardo (2011) et Pons (2015) c'est-à-dire qu'il n'existe pas d'univocité pour accepter ce terme comme un élément de la CNV, malgré le fait que cette matière concerne principalement les psychologues qui ont apporté des produits tellement représentatifs pour bien comprendre l'expression humaine (Cosnier. 1996).

Plusieurs de ces recherches significatives sont proposées par la psychologie évolutionniste, en expliquant par une théorie de type Darwinienne (De Waal, 2002; Bacáicoa, 2006), les effets immédiats de la mesure, de la forme et de la proportion du corps², dans le choix du partenaire (Lecalennier, 2007), la valorisation de l'attractivité (Abetz

1 Cela a été choisi comme méthode de l'ontologie élémentale, en accueillant également les dispositions de François Rastier (1998) et Ricœur (2006) en trois axes a) la création d'un cadre sémantique de base, b) l'identification des éléments, les caractéristiques et les catégories structurelles des concepts, c) le contraste objectif du FA dans la CNV.

2 Recherches de type anthropomorphologique, un terme qui fait référence à la discipline scientifique qui se spécialise dans l'étude des formes et des proportions du corps, de plus son cadre théorique comprend le développement de l'analyse anthropométrique, qui se réfère à l'étude spécialisée des mesures du corps. 
En 2002, Vera França, dans son écrit "Paradigmas da comunicaçâo: conhecer o quê?" précise le manque de diligence et d'intérêt pour développer un cadre théorique dans la communication, car il n'y a pas de critères unifiés qui délimitent ce domaine de la connaissance, sur cette base, il est urgent de produire un accord minimum dans la communauté scientifique. et Wade, 1997), l'investissement parental (Buss, 2016) lors de l'évaluation lieder (Little et Robert, 2012) ou dans la conduite de fuite (Daly et Wilson, 1999). Malgré le fait que les résultats pré mentionnés aient une portée psychobiologique, ceux-ci sont contenus dans le domaine de la cognition sociale, par conséquent, bien qu'ils n'aient pas évalués son importance dans l'étude spécifique de l'expression non verbale, c'est pour cette raison que les résultats sont pertinents, car ils expliquent leurs mécanismes dans l'interaction entre les êtres humains, raison pour laquelle il est nécessaire de réaliser une méta-analyse de ces contributions depuis la perspective de la communication.

À première vue dans toutes les productions scientifiques mentionnées, nous pouvons constater qu'il y a une relation entre les gens, où le cerveau d'un sujet reçoit des informations du stimule (corps) d'un autre participant et avec la base sur ce dernier, le premier génère une réponse; tout se passe sans un mot, en accomplissant de cette façon les caractéristiques de la CNV. Cependant, il existe une autre position, dont l'argument est que ce processus peut être bien expliqué par la théorie perceptive de Goldstein (2009), en comprenant que le FA est comparable au décodage d'un objet quotidien, sans que cela implique la vocation communicative.

Par conséquent, la question se pose dans cette recherche: est-ce que le facteur anthropomorphe est un élément d'analyse dans la communication non verbale? La forme du corps exprime-t-elle un message susceptible d'être interprété? Quelles études en anthropomorphologie faites par les psychologues évolutionnistes peuvent être appliquées à la compréhension de la communication non verbale?

Pour répondre à ces questions, l'objectif principal est d'élucider si le facteur anthropomorphe est un élément d'analyse dans la communication non verbale. De manière secondaire, il est proposé d'identifier l'ontologie de ces deux concepts, ce qui permet d'étudier si la forme, la proportion et les mesures du corps expriment un message, tout cela en examinant les contributions de la psychologie évolutionniste dans ce domaine.

\section{Ontologie de la communication non verbale et le facteur an- thropomorphe}

\section{Ontologie de la communication non verbale}

En 2002, Vera França, dans son écrit "Paradigmas da comunicaçâo: conhecer o quê?" précise le manque de diligence et d'intérêt pour développer un cadre théorique dans la communication, car il n'y a pas de critères unifiés qui délimitent ce domaine de la connaissance, sur cette base, il est urgent de produire un accord minimum dans la communauté scientifique (Rodríguez, 2015).

Étant donné qu'il n'existe pas d'univocité, il est donc nécessaire d'invoquer les racines sémantiques du concept. Ainsi, étymologiquement selon la Société Académique pour l'Étude des Langues Romances (SAELR) (2009), la communication non verbale se compose du latin "communicatio" qui signifie action ou effet de transmettre des messages" (p.147), "Non: nulle chose" (p.345) et verbale "verbalis: relative aux mots" (p.563). En intégrant les facteurs, cela se définirait comme l'acte de transmettre des messages sans utiliser les mots.

Cependant, de manière générique à la communication "nous ne pouvons pas la réduire au simple fait "de transmettre l'information" (Duarte, 2003, p. 52), mais cela 
implique "l'activité de l'articulation à travers laquelle, les participants socialement définis, négocient des significations concrètes dans un scénario physique, temporel et social" (Chi-yue et Qiu, 2014, p.1, notre traduction). Par conséquent, Henriquez, (1971) cité dans (Croussy, 1989, p.33), le définie comme le "Processus par lequel une source d'informations $A$ tend à agir sur un récepteur d'informations $B$ de manière à provoquer chez celui-ci l'apparition d'actes ou de sentiments permettant une régulation des activités de B ou du groupe auquel appartiennent A et B".

Sur cette base, Spielmann (2011) accueille les éléments basiques que possède tout acte de communication et par conséquent, la forme d'expression non verbale accomplit strictement chacun de ces composants, tel que le démontre le Tableau suivant:

Tableau 1. Schéma descriptif de la CNV dans les éléments généraux de la communication

\begin{tabular}{lcc}
\hline Élément & \multicolumn{1}{c}{ Description } & $\begin{array}{c}\text { Exemples explicatifs } \\
\text { (non énonciatifs) }\end{array}$ \\
\hline 1.Le binôme & L'existence de deux partis un émetteur et un récepteur & Toutes les personnes \\
2. Le code & Le système par lequel le message est transmis & Tous les signes non linguistiques \\
2.1 Canal & Moyens par lequel l'information est transmise. & Visuel, auditif, kinesthésique (tactile- \\
odorat)
\end{tabular}

Les exemples et les définitions sont traduits de Rodríguez (2015, p.5)

Le Tableau 1 est une vérification d'éléments, ce qui permet de comprendre que la définition la plus précise de la communication non verbale soit: le processus entre deux parties de partager tout type de messages par un moyen différent aux mots, en exprimant des informations à travers un code non linguistique, à savoir, en utilisant la diversité des signes, symboles ou d'autres stimulus de cette magnitude dépourvus de syntaxe, dont le résultat est un décodage psychologique et une réponse immédiate du récepteur (Rodríguez, 2015). Par rapport à ce qui précède, il est préférable d'utiliser le terme "partie" d'abord parce qu'il peut accueillir une pluralité de participants, d'autre part, selon Korpikiewicz (2004) une caractéristique structurelle de la CNV serait qu'elle fait partie de plusieurs espèces du règne animal.

Ainsi, plusieurs auteurs insistent que la communication non linguistique, du fait qu'elle fait partie d'autres groupes taxonomiques, possède un autre principe inhérent: la spontanéité, ce qui implique que son exécution n'intervient pas par la conscience, tout du moins pas dans une grande partie du processus (Little et Robert. 2012), Elle procède grâce à un lien entre l'émission d'information non-verbale et les circuits neuronaux responsables des instincts (Buss, 2016), particulièrement l'expression d'émotions, laquelle est activée de façon autonome par la biologie humaine, par conséquent, la CNV partage une grande partie de cette architecture cérébrale, en l'utilisant pour coder ou décoder les messages (Daly et Wilson, 1999). 
En outre, il est impératif de noter que cette forme de communication non-linguistique, ne va pas de manière indépendante du réseau verbal, mais "est utile pour soutenir ce système" (Mc. Entee, 2004, p.41, notre traduction), pour cette raison, elle remplit certaines fonctions affirmées par Paul Ekman (1965, cité par Mc. Entee, 2004, p.41) comme nous pouvons le voir dans le schéma suivant:

Tableau 2. Les fonctions de la communication non verbale

\begin{tabular}{ll}
\hline Fonction & \multicolumn{1}{c}{ Description } \\
\hline Répétition & Retransmettre le message envoyé par un code linguistique, mais de manière non verbale. \\
Remplacement & Transmettre un message par un code non verbal en pouvant être envoyé à travers un code linguistique. \\
Contradiction & $\begin{array}{l}\text { Envoyer via un code non verbal un message au sens opposé à l'information envoyée à travers les } \\
\text { mots. }\end{array}$ \\
Complément & Ajoute ou agrandit le message verbal. \\
Accentuer & Souligne ou intensifie certaines parties du message verbal. \\
Régler ou contrôler & $\begin{array}{l}\text { Mesure le flux de la conversation ou indique s'il existe des problèmes dans la réception du message } \\
\text { envoyé par un code verbal. }\end{array}$ \\
\hline
\end{tabular}

Les exemples et les définitions sont traduits du travail de Rodríguez (2015, p.5).

Concernant les fonctions du tableau précédent, Hargie (2011) ajoute que celles-ci se sont en rapport avec le réseau linguistique à travers deux taches fondamentales. La première, permet d'appuyer la construction d'un signifié et la seconde, de réfuter le contenu verbal par le biais de l'envoi de messages opposés, de sorte qu'il est nécessaire de vérifier ces conditions pour la correcte herméneutique du processus communicatif.

\section{Ontologie du facteur anthropomorphe}

Selon SAELR (2009), le mot "anthropomorphe" vient du Grecque "anthrōpó" dont la signification est "l'homme ou l'être humain" (p.25) et "morphos" qui se traduit en "la forme, la figure ou l'apparence" (p.201). Par ailleurs, ce dernier se définit comme "1.Configuration externe, 2. Dimensions ou 3. Périmètre de quelque chose" (p.201). L'intégration des facteurs entraînerait comme résultat que le mot anthropomorphe renvoie à la forme, la figure ou l'apparence de l'homme.

Plusieurs des terminologies ci-dessus mentionnées dans les définitions, ont été développées par la psychologie évolutionniste, en ayant comme catégories d'analyse: la forme (Faucher et Poirier 2010), l'apparence (Clavien al., 2007), les mesures (Rhodes, 2006) et les proportions du corps (Buss, 2016). Cependant, l'interaction entre ceux-ci tend à les joindre en tant qu'éléments dérivés: la taille, le volume corporel, la couleur et la texture apparente de la peau (Little et Robert., 2012).

\section{Information provenant du facteur anthropomorphe: études de la psychologie évolutionniste Évaluation des mesures, formes et proportions}

\section{Études anthropométriques: Symétrie}

La symétrie fait référence au fait que si un objet se divisait en deux parties, les mesures des deux moitiés sont les mêmes (Casans, 2001), sur cet aspect, plusieurs études ont été réalisées pour montrer qu'il y a des éléments inconscients qui font 
Pág 132

Ainsi, d'autres trouvailles qui utilisent des photos réelles comme méthode de travail, ont démontré qu'une haute asymétrie faciale, évaluée par plusieurs groupes de personnes, est liée aux maladies et cela ferait donc réduire l'attirance (Thornhill et Gangestad, 2006), l'opposé se déroule avec d'autres recherches qui ont trouvé des corrélations positives entre la symétrie et les gens qui la lient avec une bonne condition physique, ce qui augmente le score d'attirance. percevoir une personne comme belle-attirante, c'est-à-dire que dans le monde entier, il existe caractéristiques que les hommes et les femmes trouvent plus ou moins attirantes (Little et al., 2011; Buss, 2016).

Sur cette thèse, les contributions académiques signalent qu'il y a de multiples prémisses pour qu'un visage résulte plus attirant, une d'entre elles correspond à la proportion d'or ou de numéro phi (Castro, Lazzari, Moresca, Petrelli, et Rodrigues, 2012), ce qui explique le lien qui existe entre deux segments de la même ligne, en utilisant une opération mathématique qui est: $((a+b) / a=a / b)$ où ( $a$ ) est le segment avec la plus grande mesure et (b) est le segment avec la plus petite mesure, le produit de cette opération doit être "1.618", décimal correspondant au numéro d'or ou phi (Casans, 2001, p.11, notre traduction).

Pour effectuer cette analyse, certaines études ont pris comme référence les lignes de contour du visage (front, nez, sourcils, lèvres, joues, etc.) Ainsi, plus les segments du visage sont proches par rapport au numéro d'or, plus le visage est attirant et symétrique. Par ailleurs, cette relation peut également être trouvée dans d'autres parties du corps humain (les mains, les jambes, les bras, le torse, le dos), sur cette base, on peut en déduire que le cerveau est capable de faire ces calculs inconsciemment (Perrett et al., 1999; Casans, 2001).

D'autres recherches, développent des travaux sophistiqués de manipulation directe d'images faciales d'êtres humains, où à partir d'une photographie originale, en utilisant un programme spécialisé, on génère une version en miroir des deux moitiés du visage (symétrique) et une autre méthode avec des mesures légèrement déformées, puis, des photos sont présentées à un groupe de personnes pour qu'elles choisissent quel visage semble le plus beau. Les résultats de cet exercice montrent que la symétrie est préférée lors de la sélection et de plus cela se corrèle positivement avec une majeur ponctuation d'attirant (Perrett et al., 1999; Rhodes, Proffitt, Grady, et Sumich, 1998).

Ainsi, d'autres trouvailles qui utilisent des photos réelles comme méthode de travail, ont démontré qu'une haute asymétrie faciale, évaluée par plusieurs groupes de personnes, est liée aux maladies et cela ferait donc réduire l'attirance (Thornhill et Gangestad, 2006), l'opposé se déroule avec d'autres recherches qui ont trouvé des corrélations positives entre la symétrie et les gens qui la lient avec une bonne condition physique, ce qui augmente le score d'attirance (Jones et al., 2001).

\section{Proportion}

Une autre catégorie d'analyse est la proportion, entendue comme la relation, l'équilibre ou la concordance qui existe entre les composants d'un tout, ce concept est d'importance vitale et influe donc sur la perception de féminité ou de la masculinité d'une personne, laquelle, est décodée dans le cerveau, d'un côté, à travers l'estimation du volume de certaines parties du visage, par exemple: la mandibule d'une femme est plus étroite que celle d'un homme et les sourcils sont moins nombreux (Alicke, Smith, et Klotz, 1989; Buss, 2016).

Ce qui précède influe sur la sélection de partenaire, ce qui veut dire que, les hommes hétérosexuels choisissent dans une plus grande mesure les femmes avec une physionomie faciale féminine, ce qui équivaut à des silhouettes minces ou moins anguleuses (Rhodes et al., 1998), d'un autre côté, les femmes hétérosexuelles "préfèrent et cherchent des hommes de grande valeur, ce qui reflète la masculinité et la symétrie" (Buss, 2016, p.125, notre traduction), raison pour laquelle certaines carac- 
Pág 133

Les personnes évaluent aussi l'aspect physique en peu de secondes (Ambady, Bernieri, et Richeson, 2000) en premier lieu, comme un mécanisme pour réveiller l'émotion de dégoût après avoir détecté des phénotypes de parents proches et ainsi éviter l'inceste (Clavien et al., 2007), deuxièment, pour examiner la force ou la virilité, par exemple, les cernes, une couleur jaune et une texture hétérogène de la peau sont interprétées par le cerveau comme un indice de maladie, un parasitisme ou une faiblesse; des conclusions opposées sont déchiffrées de la couleur rouge qui est associée à la santé et à la vigueur téristiques sont analysées physiquement au niveau du visage: des yeux assez petits, des sourcils plus gros, le duvet facial, une largeur mandibulaire ou un soulèvement prononcé du menton, ces caractéristiques antérieures sont des propriétés qui sont décodées comme des traits de masculinité, par conséquent, du bon niveau de testostérone (Kruger, 2006; Little et Robert, 2012).

De la même manière, la forme du corps influe aussi sur l'identification de genre, ainsi, une expérience réalisée par Johnson, lida et Tassinary (2012) permet de conclure que le cerveau d'un sujet, identifie premièrement, la relation de taille entre les membres supérieurs (les épaules - le dos- les bras) vs (les hanches - la ceinture). Deuxièment, le cerveau sait que si la ceinture est plus petite et les hanches sont plus larges, les dites caractéristiques sont celles du corps d'une femme, tandis que si le dos est plus large et si la relation ceinture-hanche n'a pas de grandes différences, on est devant la silhouette d'un homme (Braun et Bryan, 2006).

\section{Évaluation de l'apparence, de la forme et son lien avec les attributs de la person- nalité}

Des études concluent que la tailles des hommes est perçue par les femmes hétérosexuelles comme une propriété de l'attrait masculin ce que l'on traduit comme un avantage pour être sélectionné en tant que compagnon de rendez-vous (Shepperd et Strathman, 1989; Hensley, 1994) ou conquérir de plus belles conquêtes (Berscheid, Dion, Walster, et Walster, 1971).

D'un autre côté, la littérature académique a aussi trouvé qu'une grande taille est un facteur pour que quelqu'un soit perçu comme une personne d'autorité ou avec un leadership (Blaker et al. 2013), par exemple, dans le football, les plus grands arbitres sont vus d'une plus grande autorité que les petits (Stulp, Buunk, Verhulst, et Pollet, 2012), cela s'explique par d'autres apports, où les hommes grands avec un dos larges sont identifiés comme plus protecteurs, avec plus de force, plus agressifs et même des leaders, de plus, ces deux propriétés physiques sont considérées via le cerveau par un individu dans une confrontation, pour savoir s'il doit fuir ou attaquer (Daly et Wilson, 1999; Buss, 2016).

Par rapport au sujet d'apparence, un autre corollaire d'articles indique que les personnes rattachent l'aspect de maturité faciale à l'expérience professionnelle et interprètent des aspects agressifs, avec le pouvoir (Wong, Ormiston, et Haselhuhn, 2011), sur cette base, il faut souligner que selon Keating, Mazur et Segall (1981) il existe des caractéristiques du visage associées à la domination dans plusieurs cultures.

D'autre part, les personnes évaluent aussi l'aspect physique en peu de secondes (Ambady, Bernieri, et Richeson, 2000) en premier lieu, comme un mécanisme pour réveiller l'émotion de dégoût après avoir détecté des phénotypes de parents proches et ainsi éviter l'inceste (Clavien et al., 2007), deuxièment, pour examiner la force ou la virilité, par exemple, les cernes, une couleur jaune et une texture hétérogène de la peau sont interprétées par le cerveau comme un indice de maladie, un parasitisme ou une faiblesse; des conclusions opposées sont déchiffrées de la couleur rouge qui est associée à la santé et à la vigueur (Little et al., 2011).

Cependant, il existe beaucoup d'autres attributs de l'apparence qui sont décodés par le cerveau et influent sur la perception de l'attrait, de l'âge, de la force, de l'origine raciale, du poids corporel et d'autres caractéristiques. Néanmoins, il y a beaucoup d'autres attributs d'apparence qui sont décodés par le cerveau et influencent à la 
Pág 134

En revanche, les cheveux maltraités réduisent l'attractivité du visage et la santé perçue3 (Mesko et Bereczki, 2004). Des informations similaires sont transmises par la couleur des cheveux, parce que dans diverses études, les blondes sont classées comme plus attrayantes, vitales, féminines, émotionnelles et sensuelles, tandis que les femmes avec les cheveux châtains, sont perçues étant plus intelligentes, tout cela en comparant les réponses des participants dans différentes gammes de couleur de cheveux. fois en ce qui est attrayant tout comme dans d'autres domaines. Le premier de ces attributs sont les cheveux, car, "plusieurs styles (courts, longs, avec des noeuds, décoiffés) influent grandement sur la valorisation attractive, en termes d'âge, de féminité et de l'intérêt sexuel" (Bereczki et Mesko, 2006, p.36, notre traduction), par exemple, les cheveux longs ou de taille moyenne, augmentent significativement l'attrait d'une femme, de plus, s'ils sont lumineux et coiffés, une plus grande vitalité est transmise ainsi qu'un certain effet de jeunesse. En revanche, les cheveux maltraités réduisent l'attractivité du visage et la santé perçue ${ }^{3}$ (Mesko et Bereczki, 2004). Des informations similaires sont transmises par la couleur des cheveux, parce que dans diverses études, les blondes sont classées comme plus attrayantes, vitales, féminines, émotionnelles et sensuelles, tandis que les femmes avec les cheveux châtains, sont perçues étant plus intelligentes, tout cela en comparant les réponses des participants dans différentes gammes de couleur de cheveux (Mesko et Bereczki, 2004).

En second terme on retrouve la barbe, laquelle affecte la valorisation d'attributs socio-sexuelles, sur cet aspect, des trouvailles de Dixson y Brooks (2013) suggèrent qu'un niveau intermédiaire de duvet facial est interprété comme étant plus attractif, tandis que, les hommes avec une barbe complète ${ }^{4}$ son perçus comme des meilleurs pères, avec une meilleur capacité à protéger et avec de bonnes qualités génétiques pour procréer ${ }^{5}$. Additionnellement, d'autres recherches démontrent que la barbe influe en augmentant la perception de masculinité, de maturité, de dominance, et d'agressivité (Archer, 2009; Dixson et Vasey, 2012), l'effet contraire se reproduit avec des personnes totalement rasées, puisque ces éléments sont associés à la jeunesse et à l'accessibilité.

En troisième lieu, il y a les éléments corporels suivants qui permettent d'évaluer le degré de vieillissement: la calvitie, les poches sous les yeux, l'affaissement des paupières, les rides, les cheveux gris, des taches ou la perte de fermeté et l'éclat de la peau. En dernier terme, plusieurs aspects qui envoient une information sociale, culturelle ou qui sont associés à des stéréotypes (Little et Robert, 2012), telles que les différentes couleurs de peau ou d'autres phénotypes indiquant le groupe ethnique auquel une personne appartient (noir, caucasien, indo-aryen, arabe, Indiens d'Amérique, métisses, etc.) (Keating et al., 1981).

\section{Le facteur anthropomorphe un élément d'analyse dans la communication non verbale}

Les recherches antérieurement décrites permettent de déduire que dans l'interaction de deux humains, le cerveau de l'un d'eux par rapport à l'autre, décode une information sur le stimule "corps" (dans ses différentes catégories d'analyse), lui assigne un signifié, et en relation avec cela, le cerveau génère une réponse immédiate, qui peut être un acte (par exemple parler à un futur conjoint) ou un sentiment (un dégoût), ces éléments satisfont la définition de Henríquez, (1971, cité dans Croussy, 1989), de plus, cela respecte de manière stricte tous les éléments nécessaires pour qu'un acte communicatif existe, tel que cela se démontre dans le Tableau 3 , en honorant ainsi ce que stipule Spielmann (2011).

Comme le montre le Tableau 3, ce qui est transmis par l'apparence, les mesures, les formes, les proportions du corps et de ses dérivés, tout cela ne se fait pas à travers des mots, mais pour cela, tout s'accomplit avec la définition étymologique de la CNV,

3 Effet qui est aussi partagé par le genre masculin.

4 Le terme fait référence au fait que les poils du visage sont uniformes, denses et recouvrent la totalité de la zone de la barbe, en ne laissant aucun espace à couvrir.

5 Conclusion dérivée de la comparaison entre : la barbe complète vs celle de niveau moyen, complète vs incomplètes, complète vs absence de poils au visage. 
Tableau 3. Le Facteur anthropomorphe dans les éléments de l'acte communicatif

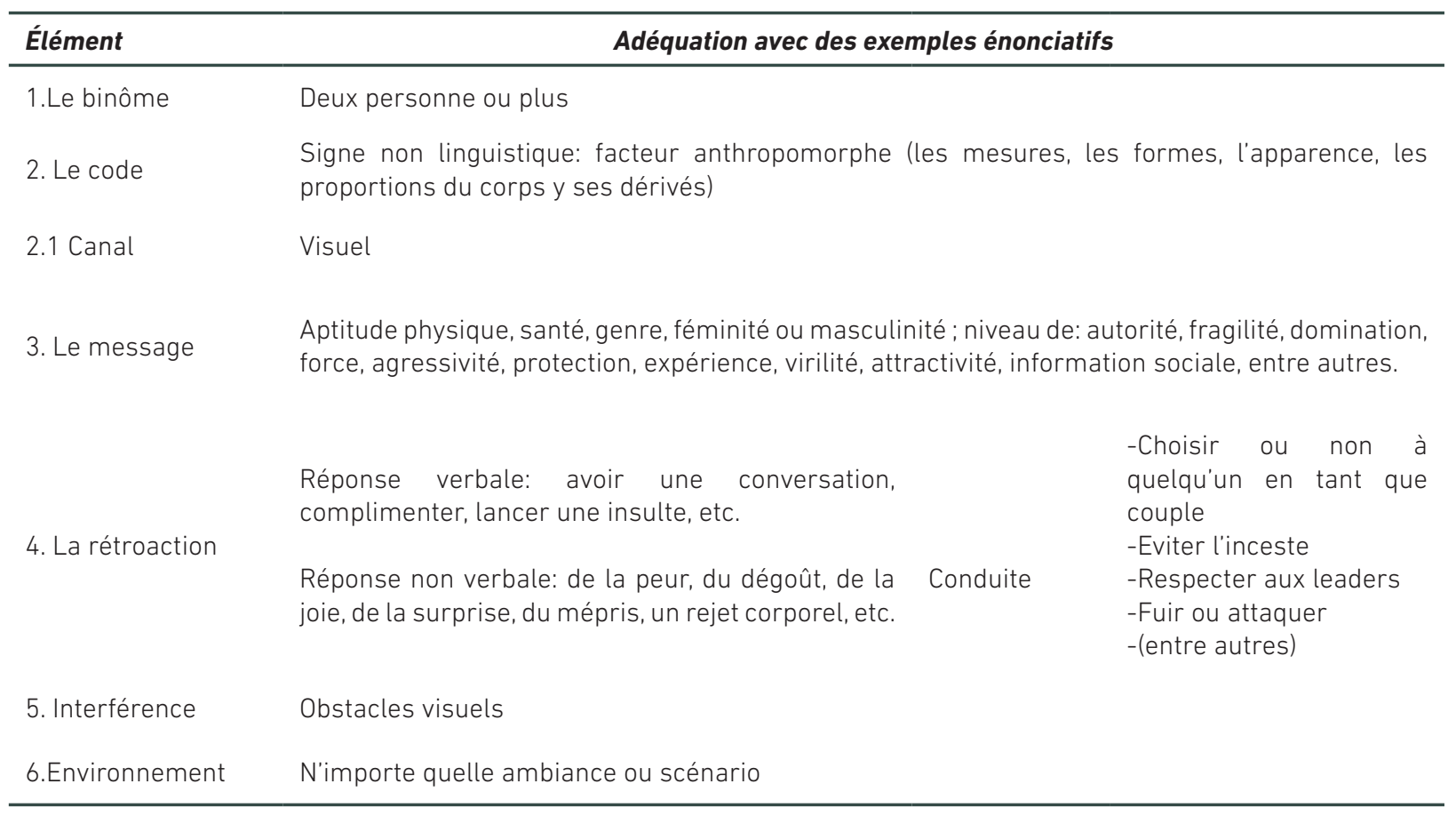

Le schéma ci-dessus possède des exemples qui ont été choisis par nos soins

la colonne de l'étude présente. De plus, de la même manière l'information provenant du FA, est interprétable, celle-ci s'élève à la catégorie de message, étant donné qu'il existe pour une raison, le message n'est pas réalisé d'une forme concise, ni dépourvu du sens (Vigotsky, 1962), sinon que son signifié, avec des caractéristiques uniformes, peut produire une grande variété de réponses, dans certains cas, en dépendant des préférences, des goûts, de la personnalité ou d'autre aspect de la cognition psychologique du récepteur (Richard et al., 1990; Bernard, 2015).

Malgré ce qui est mentionné antérieurement, il est indispensable d'examiner que pour quelques auteurs, il doit y avoir une intention d'exprimer ou d'exécuter le codage conscient d'un message (Chi-yue et Qiu, 2014), sous peine que cela soit un simple acte de sens-perception, cependant, en termes techniques il n'existe pas toujours cette vocation communicative, de façon à ce que, parfois, comme cela arrive avec le langage corporel et spécialement avec les micro-expressions, les mouvements musculaires apparaissent sans la volonté de l'émetteur, de sorte que, par cette voie, la configuration physique du corps peut aussi être considérée comme appartenant au processus de communication.

Le facteur anthropomorphe et la communication non verbale dans le règne animal Une autre caractéristique de la CNV selon Honorata Korpikiewicz est qu'elle "est innée et amplement utilisée par tous les animaux" (2004, p.14) c'est-à-dire qu'elle n'est pas exclusive aux humains, de cette façon, le FA est possible de se retrouver comme un mécanisme d'expressions dans d'autres espèces, par exemple, certains papillons femelles choisissent leurs mâles en vertu de leurs couleurs, des dessins et des patrons de leurs ailes, pour ensuite copuler. Un cas concret du type Bicyclus anynana, qui utilise des taches réfléchissantes avec une forme d'œil localisées dans la partie supérieure de l'aile, pour attirer les futures partenaires d'accouplement (Robertson et Monteiro 2005). 
Pág 136

Ces enquêtes soutiennent la thèse que le FA est un outil d'expression non verbale présente dans le règne animal, par conséquent, il est possible de l'envisager comme un élément d'analyse pour les relations sociales, depuis le point de vue de la psychologie comparée.
En outre, il existe des preuves solides pour conclure que d'autres insectes se servent également de cette partie du corps pour simuler qu'ils sont plus grands, en communiquant de la force, de la supériorité et même de la domination, afin d'effrayer des prédateurs ou des opposants (McMillan, Monteiro, et Kapan, 2002). Ensuite, ce qui précède montre qu'il y a un usage semblable de l'information du FA entre ces invertébrés et l'homo sapiens sapiens, en termes de choix d'un partenaire ou de la conduite de fuite (Daly et Wilson 1999; Maestripieri, 2012).

Cependant, en ce qui concerne l'apparence, Little et al. (2011) donnent à penser que la couleur est également importante dans "la sélection sexuelle de plusieurs espèces de poissons, d'oiseaux et d'autres mammifères non humains" (p.1643, notre traduction). Ainsi, en parlant des types taxonomiques plus proches, il existe des anthropoïdes comme le Mandrillus Sphinx, qui évaluent en primates masculins, une coloration rouge saturée sur le visage, sur les fesses et les organes génitaux, comme un signe d'une grande capacité de lutte, de domination et même d'estatus (Setchell et Wickings, 2005).

À son tour, en ce qui concerne l'évaluation de l'attirance dans l'ordre des primates, le sexe féminin juge les tailles, les formes et les proportions du corps des mâles, afin de s'accoupler avec ceux qui montrent des signes des meilleures conditions de santé, de force physique ou de virilité, et elles sont même en mesure d'évaluer les traits physionomiques pour éviter de copuler avec d'autres primates possédant de lien de parenté (Dixson, 2012), cette situation est similaire au mécanisme humain qui vise à prévenir l'inceste (Clavien et al., 2007).

Ces enquêtes soutiennent la thèse que le FA est un outil d'expression non verbale présente dans le règne animal, par conséquent, il est possible de l'envisager comme un élément d'analyse pour les relations sociales, depuis le point de vue de la psychologie comparéeb.

\section{Application du facteur anthropomorphe dans les fonctions de la communication non verbale}

En parlant d'un autre aspect ontologique, la communication par codes non-linguistiques sert comme un soutien interdépendant du système verbal (Mc. Entee, 2004) par le biais de six fonctions développées dans le Tableau 2, de sorte que, en analysant si le FA les traite, il est démontré que la fonction de répétition peut être effectuée de manière exemplifiée, si d'une part, une personne à travers les mots dit: "Je suis fort" et que sa forme du corps laisse voir une masse corporelle musclée, à travers ce que fournit Daly et Wilson (1999), il est connu que le volume du corps est associé à la perception de la force, ainsi il revient à transmettre le même message, par deux codes.

Pour sa part, le travail de remplacement, peut se voir en évidence de manière quotidienne, en prenant comme base les trouvailles de Johnson et al. (2012) en somme, les gens ne doivent pas dire leur sexe "mâle-femelle" car cela est interprété par le cerveau, en premier lieu, en évaluant des portions de la partie supérieure du corps en relation aux hanches et à la ceinture, en deuxième lieu, en analysant des éléments tels que le duvet facial et une large mâchoire (Little et Robert, 2012). Donc, de cette manière il est transmis de manière non-linguistique l'information qui pourrait être envoyée à travers le langage articulé.

6 Forrester, Quaresmini, Leavens, Mareschal, et Thomas (2013) et (Goldberg, 2010), argumentent que les animaux comme les humains utilisent des habiletés non verbales afin de générer la cohésion sociale, ainsi, "l'étude des espèces roches a l'homme, permet la compréhension de plusieurs processus cognitifs et comportementaux de l'être humain" (Maestripieri, 2012, p551, notre traduction) spécialement dans la communication non linguistique. 
Pág 137

De plus, l'adéquation des fonctions de répétition, de contradiction, de substitution et de complémentarité sont suffisantes pour tenir compte du FA dans l'étude de base de l'expression humaine, non seulement parce que les tâches décrites dans le Tableau 2 ne sont pas exhaustives, sinon que cela permette de conclure que les mesures, les formes et les proportions du corps, se connectent avec le système verbal par au moins 15 messages différents (voir Tableau 3), qui aident l'interlocuteur à construire un signifié plus précis et enrichit même d'autres aspects sémantiques, des tâches qu'exécutent aussi le langage kinésique, la proxémique et le paralangage.
En ce qui concerne la contradiction, elle est exécutée par exemple dans un cas hypothétique où une personne affirme verbalement être en bonne santé, mais si cette personne a la peau jaune ainsi qu'une texture hétérogène, qui sont des traits interprétés par le cerveau comme un signe de maladie ou de parasitisme selon Little et al. (2011), cela réfute le message envoyé à travers les mots.

D'autre part, si un homme dit: "Je suis attirant", mais la symétrie de son visage est assez élevée, proche de la proportion d'or, grâce à l'étude de Castro et al. (2012), on peut en déduire que son interlocuteur saura que celui qui a parlé est non seulement attirant, mais qu'il l'est dans un degré bien plus élevé, élargissant ainsi le message linguistique, en ajoutant plus d'informations à ce qui est exprimé de forme verbale, par conséquent, la fonction de complément est accomplie.

Un élément crucial qui exige une observation plus profonde est la modification du corps conscient, alors, celle-ci conduit la volonté immergée d'un individu pour coder les informations, en adhérant ou en changeant leur physionomie, de sorte que, à travers celle-là, elle puisse intensifier ou potentialiser un message, qui peut être: la rébellion, la sensualité, la non-conformité, etc., ce qui implique un lien possible avec la tâche d'accentuer (représentée sur le Tableau 2), cependant, une étude empirique est nécessaire comme les exemples précédents, pour ainsi pouvoir prouver et exister avec une certitude scientifique.

Malgré le développement casuistique des quatre premières fonctions, matérialiser l'exemplification de la tâche de régulation, est un travail peu probable, étant donné que, dans le cas de connaître des problèmes en partie du message verbal ou de mesurer le flux d'une conversation, elles assument des caractéristiques spéciales, où elles interfèrent avec des capacités métacognitives, qui impliquent l'esprit volontaire d'effectuer un codage, qui est applicable en particulier dans le domaine du langage corporel ou du paralangage.

Toutefois, cela n'implique pas d'exclure que le FA est un élément de la CNV, puisque la structure ontologique de celle-ci selon Andersen (2008) présente une base essentiellement biologique, pour cela, en ce qui concerne la transmission de messages, ils ont une "même reconnaissance universelle" (p.17), à ce sujet, Little et al. (2011) disent que la méta-analyse en anthropomorphologie "suggère que les gens partout dans le monde utilisent le même ou au moins des certains critères similaires" (p.1639, notre traduction) en jugeant les aspects dérivés du physique humain.

De plus, l'adéquation des fonctions de répétition, de contradiction, de substitution et de complémentarité, sont suffisantes pour tenir compte du FA dans l'étude de base de l'expression humaine, non seulement parce que les tâches décrites dans le Tableau 2 ne sont pas exhaustives, sinon que cela permette de conclure que les mesures, les formes et les proportions du corps, se connectent avec le système verbal par au moins 15 messages différents (voir Tableau 3), qui aident l'interlocuteur à construire un signifié plus précis et enrichit même d'autres aspects sémantiques, des tâches qu'exécutent aussi le langage kinésique, la proxémique et le paralangage.

Ainsi, en tenant compte du fait que lors de la présente discussion il a été possible de montrer qu'il existe une information non verbale provenant du FA et que celle-ci, s'intègre à tous les composants structurels, à la définition, aux éléments et même à plusieurs fonctions de la CNV. On peut écarter la thèse selon laquelle l'informations dérivée de la forme, la mesure ou la proportion du corps sont clairement un 
On conclut également que le FA est un signe non-linguistique, qui exécute chaque élément structurel pour que la communication puisse exister, chaque fois que: a) s'adapte à la définition de la CNV, b) il puisse être possible d'envoyer différents messages par son biais, c) il soit possible de le retrouver comme un mécanisme d'expression dans d'autres espèces, d) il serve d'appui au système verbal au moyen de l'exécution claire de quatre fonctions et éventuellement de l'accentuation, de sorte que, de cette manière la décodification du FA n'est pas seulement un phénomène de perception sensorielle et doit alors être inclue comme un élément d'analyse de la CNV. processus de perception sensorielle, par laquelle il est nécessaire de posséder une observation plus profonde de ce code non-linguistique, de poursuivre les recherches de manière expérimentale avec une portée circonscrite dans le domaine de la psychologie appliquée à la communication, de sorte que, de cette façon il soit possible d'explorer d'autres aspects impliqués dans la cognition social.

\section{Conclusions}

On a proposé que le terme correct accepté par la communauté scientifique devrait être "facteur anthropomorphe" (FA) et non "la forme ou l'apparence du corps" comme certains articles exposent, puisque le mot FA comme sous-catégorie de la CNV, reçoit de manière intégrale d'autres éléments (la symétrie, la taille, les proportions, le volume corporelle, entre autres) de plus, cela permet aux académiciens au niveau international d'avoir un même langage et ainsi surmonter les ambiguités produites par les diverses classifications actuelles ou leurs traductions variées.

Dans ce texte, il a été déclaré que la CNV n'a pas une concertation théorique uniforme et donc le FA non plus, raison pour laquelle une étude ontologique des deux a été réalisée. Le résultat nous permet de proposer que la définition la plus éclectique du premier terme implique le processus entre deux parties de partager tout type de messages via une voix différente aux mots, cela signifie, de pouvoir exprimer une information à travers un code non-linguistique, à savoir, en utilisant la diversité des signes, des symboles ou d'autres stimuli d'une telle ampleur dépourvus de syntaxe, dont le résultat est un décodage psychologique et une réponse immédiate du récepteur.

En ce qui concerne le FA, sur la base des études élaborées par la psychologie évolutionniste on peut résumer les catégories suivantes d'analyse: la forme, l'apparence, les dimensions et les proportions du corps, cependant, l'interaction entre ces causes est incorporée comme éléments dérivés: la taille, le volume du corps, la couleur et la texture apparente de la peau.

En examinant la littérature scientifique et plusieurs méta-analyses d'études expérimentales, il est démontré que les composants du FA produisent une information interprétable, traduite dans les 15 messages suivants: l'aptitude physique, la santé, le genre, la féminité ou la masculinité, le niveau de: l'autorité, la faiblesse, la domination, la force, l'agressivité, la protection, l'expérience, la virilité, l'attraction, qui génèrent des réponses multiples, certains d'entre elles peuvent être comportementales, par exemple, choisir ou non une personne pour former un couple, éviter l'inceste, respecter l'ordre, fuir ou attaquer.

On conclut également que le FA est un signe non-linguistique, qui exécute chaque élément structurel pour que la communication puisse exister, chaque fois que: a) s'adapte à la définition de la CNV, b) il puisse être possible d'envoyer différents messages par son biais, c) il soit possible de le retrouver comme un mécanisme d'expression dans d'autres espèces, $d$ ) il serve d'appui au système verbal au moyen de l'exécution claire de quatre fonctions et éventuellement de l'accentuation, de sorte que, de cette manière la décodification du FA n'est pas seulement un phénomène de perception sensorielle et doit alors être inclue comme un élément d'analyse de la CNV.

Enfin, il est nécessaire d'avoir une observance profonde de ce code non-linguistique puisqu'actuellement dans divers écrits il est seulement énonce comme "la forme du corps et de l'apparence", dans d'autres il n'est même pas envisagé, et donc, cet article donne les bases pour poursuivre les recherches de type expérimentale avec 
une portée circonscrite dans le domaine de la psychologie appliquée à la communication, pour ainsi, de cette façon il puisse être possible d'explorer d'autres aspects du FA impliqués dans la cognition sociale, par exemple, examiner la modification du corps conscient comme forme pour coder un message et sa possible relation avec la fonction d'accentuer.

\section{Références}

Abetz, H., et Wade, J. (1997). Social Cognition and Evolutionary Psychology: Physical Attractiveness and Contrast Effects on Women's Self-perceived Body Image, International Journal of Psychology, 32(1), 35-42. http://dx.doi. org/10.1080/002075997400953

Alicke, M., Smith, R., et Klotz, M. (1989). Judgments of Physical Attractiveness: The Role of Faces and bodies, Personality and Social Psychology Bulletin, 12(4), 381 389. http://dx.doi.org/10.1177/0146167286124001

Ambady, N., Bernieri, F.J., et Richeson, J.A. (2000). Toward a histology of social behavior: Judgmental accuracy from thin slices of the behavioral stream. Advances in Experimental Social Psychology, 32(1), 201-271. http://dx.doi.org/10.1016/S00652601(00)80006-4

Andersen, P.A. (2008). Nonverbal Communication: Forms and Functions. Illinois, United States: Waveland Press.

Archer, J. (2009). Does sexual selection explain human sex differences in aggression?, Behavioral and brain sciences, 32(3), 249-266. http://doi.org/10.1017/ $\underline{\text { S0140525X09990951 }}$

Bacáicoa, F. (2006). Psicología evolutiva y psicología evolucionista claves para la discusión. Revista de Psicodidáctica, 11(1), 109-131. Repéré à http://www.redalyc. org/articulo.oa?id=17514747008

Bernard, F. (2015). Les théories de l'influence en communication: perspectives nord-américaines et françaises, Hermès, La Revue, 1(71), 45-57. Repéré à https:// www.cairn.info/resume.php?ID ARTICLE=HERM 0710045

Bereczki, T., et Mesko, N. (2006). Hair length, facial attractiveness, personality atribution: A multriple fitness model of hairdressing, Psychology Review, 15(1), 35-42. Repéré à http://mjesec.ffzg.hr/revija.psi/vol\%2013\%201\%202006/Bereczkei 2006 13-1.pdf

Berscheid, E., Dion, K., Walster, E., et Walster, G.W. (1971). Physical attractiveness and dating choice: A test of the matching hypothesis. Journal of Experimental and Social Psychology, 7(2), 173-189. http://dx.doi.org/10.1016/0022-1031(71)90065-5

Blaker, N.M., Rompa, I., Dessing, I.H., Vriend, A.F., Herscheberg, C., et Van Vugt, M. (2013). The height leadership advantage in men and women: Testing evolutionary psychology predictions about the perceptions of tall leaders. Group Processes \& Intergroup Relations, 16(1), 17-27. Repéré à http://journals.sagepub.com/doi/ pdf/10.1177/1368430212437211

Boutaud, J. (2004). Sémiotique et communication: Un malentendu qui a bien tourné. Hermès, La Revue, 38(1), 96-102. Repéré à http://documents.irevues.inist.fr/ handle/2042/9431

Braun, M. F., et Bryan, A. (2006), Female waist-to-hip and male waist-to-shoulder ratios as determinants of romantic partner desirability. Journal of Social and Personal Relationships, 23(5), 805-819. Repéré à http://www.unm.edu/ abryan/ articles/femalehipratio.pdf

Buss, D. (2016). Evolutionary psychology: The new science of mind. New York, United States: Routhledge.

Casans, A. (2001). Aspectos estéticos de la divina proporción, (thèse en doctorat, Universidad Complutense de Madrid). Repéré à http://biblioteca.ucm.es/tesis/fs// ucm-t25388.pdf. 
Casablanca, L., et Chacón, P. (2014). La moda como lenguaje: una comunicación no verbal. Revista de la Asociación Aragonesa de Arte, 29(2), 1-10. Repéré à http:// www.aacadigital.com/contenido.php?idarticulo $=1030$

Castro I., Lazzari, A.P., Moresca, R., Petrelli, E., et Rodrigues, K. (2012). Photometric study of divine proportion and its correlation with facial attractiveness. Dental Press Journal, 17(2), 1-22. http://dx.doi.org/10.1590/S2176-94512012000200022

Chi-yue, C., et Qiu, L. (2014). Communication and culture: A complexity theory approach. Asian Journal of Social Psychology, 17(1), 108-111. http://dx.doi. org/10.1111/ajsp.12054

Clavien, C. J., El-Bez, C., Berthoud, A., Clément F., Dekeuwer C., Deonna, J., . . Perrin, N. (2007). Moral et evolution biologique: entre déterminisme et liberté. Laussanne, Suisse: Presses Polythechniques et Universitaires Rommandes.

Cosnier, J. (1996). Le geste du dialogue, La communication non verbale. Repéré à http://icar.univ-lyon2.fr/membres/jcosnier/articles/ll-9 Les gestes communication.pdf

Croussy, G. (1989). La génération de la communication. Lille, France: Presses Universitaires de Lille.

Daly, M., et Wilson, M. (1999). Human evolutionary psychology and animal behavior. Animal Behaviour, 57(1), 509-519. http://dx.doi.org/10.1006/anbe.1998.1027

De Lavergne, C. (2010). La communication non verbale. Repéré à http://www.univmontp3.fr/infocom/wp-content/REC-La-communication-non-verbale2.pdf

De Waal, F. (2002). Evolutionary psychology: The wheat and chaff. American Psychological Society, 11(6), 187-191. Repéré à http://www.emory.edu/LIVING LINKS/ publications/articles/deWaal 2002.pdf

Dixson, A. F. (2012). Primate Sexuality: Comparative Studies of the Prosimians, Monkeys, Apes, and humans. (2e ed.). New York, United States: Oxford.

Dixson, B.J., et Vasey, P.L. (2012). Beards augment perceptions of men's age, social status, and aggressiveness, but not attractiveness. Behavioral Ecology, 23(3), 481-490. https://doi.org/10.1093/beheco/arr214

Dixson, B.J., et Brooks, R.C. (2013). The role of facial hair in women's perceptions of men's attractiveness, health, masculinity and parenting abilities. Evolution and Human Behavior, 34(2), 236-241. http://dx.doi.org/10.1016/j.evolhumbehav.2013.02.003

Duarte, E. (2003). Por uma epistemologia da comunicação. Dans Lopes, M. I. V. (dir.), Epistemologia da comunicaçâo (2éd., vol. 1, p. 41-54). Sâo Paulo, Brésil: Loyola.

Fast, J. (1979), El lenguaje del cuerpo. Barcelona, España: Kairos.

Faucher, L., et Poirier P. (2010). Psychologie évolutionniste et théories interdomaines. Dialogue, 40(03), 453-486. https://doi.org/10.1017/S0012217300018874

França, V. (2002). Paradigmas da comunicaçâo: conhecer o quê?. Dans Motta, L. G.; Weber, M. H.; França, V. et Paiva, R. (dir.), Estratégias e culturas da comunicaçâo (3e éd., vol. 2, p. 13-29). Brasil, Brasilia: Editora Universidade de Brasilia.

Forrester, G., Quaresmini, C., Leavens, D., Mareschal, D., et Thomas, M. (2013). Human handedness: An inherited evolutionary trait. Behavioral Brain Research, 237(1), 200-206. http://dx.doi.org/10.1016/j.bbr.2012.09.037

Garcia, J. L. (2000). Comunicación no verbal, periodismo y medios audiovisuales. Madrid, España: Universitas.

Goldberg, J. (2010). Ethologie animale et humaine. Paris, France: Frison-Roche.

Goldstein, B. E. (2009). Sensation \& Perception. San Francisco, United States: Thomson.

Hall, E.T. (1984). Le langage silencieux. Paris, France: Seuil.

Hargie, 0. (2011). Skilled Interpersonal Communication. New York, United States: Routledge. 
Hensley, W. E. (1994). Height as a basis for interpersonal attraction. Adolescence, 29(114), 469-474.

Hernández, M., et Rodríguez, I. (2009), Investigar en comunicación no verbal: un modelo para el análisis del comportamiento kinésico de líderes políticos y para la determinación de su significación estratégica. Enseñanza \& Teaching, 27(1), 61 94. Repéré à http://campus.usal.es/ revistas trabajo/index.php/0212-5374/ article/viewFile/6584/7150

Johnson, K. L., lida, M., et Tassinary, L. G. (2012). Person (mis)perception: Functionally biased sex categorization of bodies. Proceedings of the Royal Society, Biological Sciences, 279(1749), 4982- 4989. http://dx.doi.org/10.1098/rspb.2012.2060

Jones, B. C., Little, A. C., Penton-Voak, I. S., Tiddeman, B. P., Burt, D. M., et Perrett, D. I. (2001). Facial symmetry and judgements of apparent health: support for a 'good genes' explanation of the attractiveness symmetry relationship. Evolutionary Human behavior, 22(6), 417-429. http://dx.doi.org/10.1016/S1090-5138(01)00083$\underline{6}$

Keating, C. F., Mazur, A., et Segall, M. H. (1981). A cross-cultural exploration of physiognomic traits of dominance and happiness. Ethology and Sociobiology, 2(1), 41 48. http://dx.doi.org/10.1016/0162-3095(81)90021-2

Knapp, M. L. (1995). La comunicacion no verbal, el cuerpo y el entorno, Barcelona, España: Paidos.

Kruger, D.J. (2006). Male facial masculinity influences attributions of personality and reproductive strategy, Journal of Social and Personal Relationships, 13(4), 451463. http://dx.doi.org/10.1111/j.1475-6811.2006.00129.x

Korpikiewicz, H. (2004). Non verbal communication vs awareness, Lingua ac Communitas, 14(1), 13-19. Repéré à http://www.lingua.amu.edu.pl/Lingua 14/KORPIKIEWICZ 14.pdf

Kwon, J., Ogawa, K., Ono, E., et Miyake, Y. (2015). Detection of Nonverbal Synchronization through Phase Difference in Human Communication, PLOS ONE, 10(7), 1-15. http://dx.doi.org/10.1371/journal.pone.0133881

Lecalennier, F. (2007). Evolución \& psicología: El caso de la psicología evolucionista. Revista de neuropsicología de Chile, 2(2), 56-71. Repéré à http://www.redalyc. org/pdf/1793/179317753003.pdf

Leathers, D., et Eaves, M. H. (2016). Successfull non verbal communication: Principles and applications. New York, United States: Routledge.

Little, A.C., Jones, B.C., et DeBruine, L.M. (2011). Facial attractiveness: evolutionary based research, Philosofical Transaction of Royal society: Biological sciences, 366(1), 1638-1659. http://dx.doi.org/10.1098/rstb.2010.0404

Little, A.C., et Robert, S.C. (2012). Evolution, Appearance, and Occupational Success. Evolutionary Psychology, 10(5), 782-801. http://dx.doi. org/10.1177/147470491201000503

Maestripieri, D. (2012). Comparative Primate Psychology, repéré à http://primate. uchicago.edu/2012CPP.pdf

Mesko, N., et Bereczkei, T. (2004). Hairstyle as an adaptive means of displaying phenotypic quality, Human Nature, 15(3), 251-270. http://dx.doi.org/10.1007/s12110004-1008-6

Mc Entee, E. (2004). Comunicación Oral. México DF, México: Mc Graw-Hill.

McMillan, O., Monteiro, A., et Kapan, D. (2002). Development and evolution on the wing. Trends in Ecology and Evolution, 17(3), 125-133. http://dx.doi.org/10.1016/ s0169-5347(01)02427-2

Owen, H. (2011). Skilled Interpersonal Interaction: Research, Theory, and Practice. London, England: Routledge. 
Perrett, D. I., Burt, D. M., Penton-Voak, I. S., Lee, K.J., Rowland, D. A., et Edwards, R. (1999). Symmetry and human facial attractiveness. Evolutionary and Human Behavior, 20(5), 295-307. http://dx.doi.org/10.1016/S1090-5138(99)00014-8

Pons, C. (2015). Comunicación no verbal. Barcelona, España: kairós.

Rastier, F. (1998), Le problème épistémologique du contexte et le statut de l'interprétation dans les sciences du langage, language, 98(129), 97-111.

Richard, J., Bonnet, C., Ghiglione, R., Bromberg, M., Beauvois, J., Doise, W., et Deschamps, J. (1990). Traité de psychologie cognitive: cognition, représentation, communication. Paris, France: Dunod.

Ricœur, P. (2006). Le conflit des interprétations. Essais d'herméneutique II. Paris, France: Le Seuil.

Rhodes, A. (2006). The Evolutionary Psychology of Facial Beauty. Annual Review of Psychology, 57(1), 199-226. http://dx.doi.org/10.1146/annurev. psych.57.102904.190208

Rhodes, G., Proffitt, F., Grady, J., et Sumich, A. (1998). Facial symmetry and the perception of beauty. Psychonomic Bulletin \& Review, 5(4), 659-669. http://dx.doi. org/10.3758/BF03208842

Robertson, K.A., et Monteiro A. (2005). Female Bicyclus anynana butterflies choose males on the basis of their dorsal UV-reflective eyespot pupils. Proceedings of the Royal Society B: Biological Sciences, 272(1572), 1541-1546. http://dx.doi. org/10.1098/rspb.2005.3142

Robles, C., Hernandez, F. et Fajardo, G. (2011). La comunicación humana y su relación con la queja médica. Conamed, 16(3), 141-147.

Rodríguez, J. (2015). Aproximación al factor antropomorfo: el cuerpo como estímulo en la comunicación no verbal (thèse de license inédit). Institución Universitaria Politécnico Grancolombiano.

Setchell, J. M., et Wickings, E. J. (2005). Dominance, status signals and coloration in male mandrills (Mandrillus sphinx). Ethology, 111(1), 25-50. http://dx.doi. org/10.1111/j.1439-0310.2004.01054.x

Shepperd, J. A., et Strathman, A. J. (1989). Attractiveness and height: The role of stature in dating preference, frequency of dating, and perceptions of attractiveness. Personality and Social Psychology Bulletin, 15(4), 617-627. http://dx.doi. org/10.1177/0146167289154014

Société Académique pour l'Étude des Langue Romances (SAELR). (2009). Dictionnaire pour l'étude avancé des Langues Romances ( $2^{\mathrm{e}}$ éd., vols. 1-27), Bordeaux, France: SAELR.

Spielmann, G. (2011). Théorie(s) de la communication le modèle classique à six éléments. Repéré à http://faculty.georgetown.edu/spielmag/docs/comm/commschema.htm

Stulp, G., Buunk, A. P., Verhulst, S., et Pollet, T.V. (2012). High and mighty: Height increases authority in professional refereeing. Evolutionary Psychology, 10(1), 588-601. Repéré à http://journals.sagepub.com/doi/full/10.1177/147470491201000314

Thornhill, R., et Gangestad, S. W. (2006). Facial sexual dimorphism, developmental stability, and susceptibility to disease in men and women. Evolutionary and Human Behavior, 27(2), 131-144. http://dx.doi.org/10.1016/j.evolhumbehav.2005.06.001

Vigotsky, L.S. (1962). Thought and Language. Cambridge, England: MIT Press

Wong, E. M., Ormiston, M. E., et Haselhuhn, M. P. (2011). A face only an investor could love: CEOs' facial structure predicts their firms' financial performance. Psychological Science, 22(1), 1478-1483. http://dx.doi.org/10.1177/0956797611418838

Xu, Y., Lee, A., Wing-Li, W., Liu, X., et Birkholz, P. (2013). Human Vocal Attractiveness as Signaled by Body Size Projection. Plos One, 8(4), 1-9. http://dx.doi.org/10.1371/ journal.pone.0062397 